\title{
Proton-Pump Inhibitors Could Be Innocent When Used Concomitantly With Clopidogrel
}

\author{
Hisanori Horiuchi, MD, PhD
}

A proton-pump inhibitor (PPI) is recommended for patients with a high risk of gastrointestinal bleeding induced by nonsteroidal anti-inflammatory drugs (NSAIDs). ${ }^{1,2}$ PPIs utilize CYP2C19, a liver enzyme of cytochrome $\mathrm{P} 450$, for their inactivation, and this same enzyme mediates the generation of the active substance from the prodrug, clopidogrel, a platelet ADP-receptor blocker. ${ }^{1,2}$ Therefore, concerns have been raised that concomitant use of PPIs and clopidogrel may weaken clopidogrel's efficacy through competitive inhibition of CYP2C19 (Figure) and/or worsen the clinical outcome.

\section{Article p 2673}

In this issue of the Journal, Yano et al present their results from a randomized, open-labeled prospective study in which they found that omeprazole, a PPI, did not affect the antiplatelet function of clopidogrel or the occurrence of clinical events in patients undergoing coronary stent implantation for acute coronary syndrome (ACS) as compared with an $\mathrm{H} 2$ blocker. $^{3}$ Theirs is the first prospective study in Japanese patients with regard to this subject and provides important findings for clin-

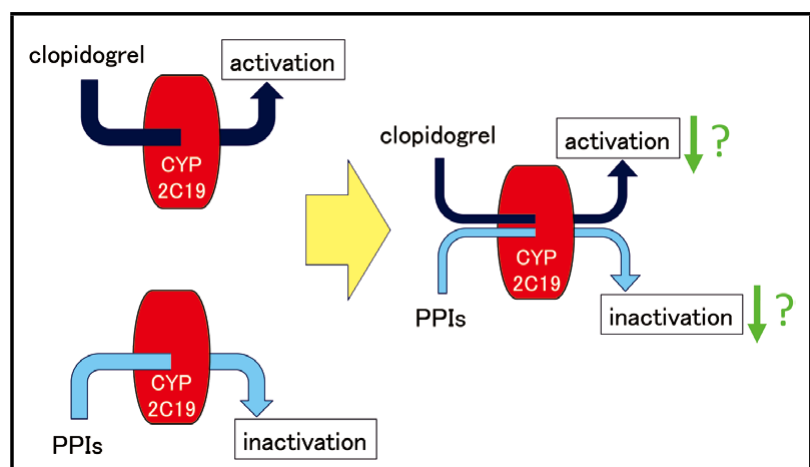

Figure. Possible competitive inhibition between clopidogrel and proton-pump inhibitors (PPIs). Clopidogrel is a prodrug that utilizes CYP2C19 for its activation, whereas PPIs utilize CYP2C19 for their inactivation. Therefore, concerns have been raised as to whether or not competitive inhibition occurs that may weaken the antiplatelet function of clopidogrel on concomitant PPI use. ical settings in Japan.

Today, more than 250,000 people per year are treated with coronary stent implantation in Japan. The most severe and notable complication is stent thrombosis and dual antiplatelet therapy (DAPT) with aspirin and an ADP-receptor blocker is routinely administered for its prevention. However, the interindividual variability of the antiplatelet effects of clopidogrel is very high and is dependent on its pharmacological properties. Clopidogrel is a prodrug that is changed into a bioactive substance through a metabolic activation process mediated by cytochrome p450 enzymes (CYPs). ${ }^{4}$ Among the CYPs, CYP2C19 has been reported to contribute most strongly to the activation of clopidogrel. The CYP2C19 gene is well known for harboring loss-of-function single nucleotide polymorphisms (SNPs) called CYP2C19*2 and CYP2C19*3. The patients with CYP2C19 SNP(s) exhibit an attenuated antiplatelet function of clopidogrel. Moreover, the CYP2C19 SNPs are associated with an increase in stent thrombosis. ${ }^{4}$ Notably, the prevalence of carriers with the SNPs in both alleles, who are referred to as poor metabolizers, is $3-5 \%$ in Caucasians and $\approx 20 \%$ in Asian populations. ${ }^{5}$ Therefore, SNPs of CYP2C19 might be a more serious problem in the clinical settings of Asian countries.

A well-known adverse effect of aspirin is gastrointestinal bleeding. In 2008, the ACCF/ACG/AHA expert consensus document for prevention of gastrointestinal bleeding under NSAID therapy was published, ${ }^{2}$ in which it was described that PPIs are recommended for high-risk bleeding patients such as those undergoing DAPT. On the other hand, it is also known that the degradation/inactivation of PPIs more or less utilizes CYP2C19. Therefore, competitive inhibition might occur when there is concomitant use of PPI and clopidogrel. Based on clinical and experimental results, ACCF/ACG/AHA revised the expert consensus in 2010 to include the consistent inhibition of clopidogrel efficacy by omeprazole, but as yet unknown effects of other PPIs because of the lack of sufficient data. ${ }^{2}$ The degree of contribution by CYP2C19 in the inactivation of each PPI is variable. Previous data suggest that the contribution of CYP2C19 is higher for omeprazole than for lansoprazole, rabeprazole and esomeprazole. ${ }^{6}$

With regard to the Japanese population, a few studies have so far been published on the pharmacological effects of concomitant use of PPIs on clopidogrel efficacy. Furuta et $\mathrm{al}^{7}$ conducted a crossover study with 39 healthy volunteers in which the antiplatelet effects were evaluated by the optical aggregom-

The opinions expressed in this article are not necessarily those of the editors or of the Japanese Circulation Society.

Received August 28, 2012; accepted August 28, 2012; released online September 19, 2012

Department of Molecular and Cellular Biology, Institute of Development, Aging and Cancer, Tohoku University, Sendai, Japan

Mailing address: Hisanori Horiuchi, MD, PhD, Department of Molecular and Cellular Biology, Institute of Development, Aging and

Cancer, Tohoku University, 4-1 Seiryomachi, Sendai 980-8575, Japan. E-mail: horiuchi@idac.tohoku.ac.jp

ISSN-1346-9843 doi:10.1253/circj.CJ-12-1117

All rights are reserved to the Japanese Circulation Society. For permissions, please e-mail: cj@j-circ.or.jp 
eter. They demonstrated that omeprazole and rabeprazole, but not lansoprazole, slightly but still significantly attenuated the antiplatelet function of clopidogrel in those without CYP2C19 SNPs, and the effects of PPIs were not detected in those with CYP2C19 SNP(s). My group also conducted a crossover study to analyze the effects of concomitant use of omeprazole or rabeprazole in 25 patients during DAPT, in which platelet reactivity was measured with the VerifyNow system. ${ }^{8}$ We reported that patients taking omeprazole exhibited slightly higher platelet reactivity than those taking rabeprazole, and that the reactivity under rabeprazole intake was similar to that detected in control patients not taking PPI. ${ }^{8}$ Thus, these studies suggest that omeprazole might significantly, although slightly, decreased the antiplatelet function of clopidogrel in the Japanese population.

In this issue of the Journal, however, Yano et al demonstrate that platelet reactivity, as evaluated by the phosphorylation levels of vasodilator-stimulated phosphoprotein (VASP), under DAPT with concomitant use of omeprazole at 14-28 days after admission for ACS was similar to that with concomitant use of famotidine, an $\mathrm{H} 2$ blocker. $^{3}$ Theoretically, famotidine is not metabolized by CYP2C19, and our study also demonstrated that the addition of famotidine did not affect the antiplatelet effect of clopidogrel. ${ }^{8}$ Therefore, these findings strongly suggest that omeprazole does not affect clopidogrel efficacy. The larger number of patients analyzed in this study $(n=138)$ would support the reliability of their results more strongly than the 2 aforementioned studies. ${ }^{7,8}$ However, the time points for evaluation of platelet reactivity were between 2 and 4 weeks after stent implantation in ACS patients, a time when platelet reactivity is elevated. The effects of ACS on platelet reactivity might have masked the effects of the drugs.

If PPIs affect the antiplatelet function of clopidogrel, they may adversely affect the prognosis of patients after stent implantation. The prospective randomized Cogent Study that was conducted in the USA recently showed that omeprazole reduced gastrointestinal events without increasing cardiovascular events. ${ }^{9}$ Furthermore, the TIMI38/TIMI44 trials demonstrated that concomitant use of omeprazole did not increase cardiovascular events, although it increased the number of patients classified as having 'clopidogrel resistance'. ${ }^{10}$ Thus, omeprazole does not seem to increase cardiovascular events in patients under DAPT in Western countries. The study by Yano et $\mathrm{al}^{3}$ reports results from a study that is, as far as I know, the first prospective randomized study for Japanese patients regarding this issue. During a follow-up period of 12 months, adverse cardiovascular events, including death from cardiovascular causes, spontaneous myocardial infarction, unstable angina, stent thrombosis, target vessel revascularization, nontarget lesion revascularization, and ischemic stroke, occurred in $13 \%$ of patients in the omeprazole group and $17 \%$ in the famotidine group. The incidences were not statistically different. It could be because the number of patients enrolled in the study was small; 138 patients were divided into the omeprazole and famotidine groups. The hard endpoints of death from cardiovascular causes, myocardial infarction or stent thrombosis did not occur in the study. ${ }^{3}$ Therefore, it seems somewhat premature to conclude from this study alone that PPIs have no influence on cardiovascular endpoints. However, 2 observational studies have recently been performed in Japan, and both also showed that cardiovascular events in patients under clopidogrel intake occurred similarly with or without PPI intake. ${ }^{11,12}$ Collectively, concomitant use of PPIs with clopidogrel does not seem to increase cardiovascular risk in the Japanese population.

In summary, the report by Yano et al in this issue of the Journal suggests that concomitant use of omeprazole with clopidogrel does not affect the antiplatelet function of clopidogrel and that omeprazole had no effect on clinical outcomes during 12 months following stent implantation in ACS patients. ${ }^{3}$ Their data must provide important information for the clinical setting, especially in Japan.

\section{Disclosures}

Conflict of Interest: None.

\section{References}

1. Bhatt DL, Scheiman J, Abraham NS, Antman EM, Chan FK, Furberg $\mathrm{CD}$, et al. ACCF/ACG/AHA 2008 expert consensus document on reducing the gastrointestinal risks of antiplatelet therapy and NSAID use: A report of the American College of Cardiology Foundation Task Force on Clinical Expert Consensus Documents. Circulation 2008; 118: $1894-1909$.

2. Abraham NS, Hlatky MA, Antman EM, Bhatt DL, Bjorkman DJ, Clark $\mathrm{CB}$, et al. ACCF/ACG/AHA 2010 expert consensus document on the concomitant use of proton pump inhibitors and thienopyridines: A focused update of the ACCF/ACG/AHA 2008 expert consensus document on reducing the gastrointestinal risks of antiplatelet therapy and NSAID use: A report of the American College of Cardiology Foundation Task Force on Expert Consensus Documents. Circulation 2010; 122: 2619-2633.

3. Yano H, Tsukahara K, Morita S, Endo T, Sugano T, Hibi K, et al. Influence of omeprazole and famotidine on the antiplatelet effects of clopidogrel in addition to aspirin in patients with acute coronary syndromes: A prospective, randomized, multicenter study. Circ J 2012; 76: $2673-2680$.

4. Mega JL, Close SL, Wiviott SD, Shen L, Hockett RD, Brandt JT, et al. Cytochrome p-450 polymorphisms and response to clopidogrel. $N$ Engl J Med 2009; 360: 354-362.

5. Desta Z, Zhao X, Shin JG, Flockhart DA. Clinical significance of the cytochrome p450 2c19 genetic polymorphism. Clin Pharmacokinet 2002; 41: $913-958$.

6. Li XQ, Andersson TB, Ahlstrom M, Weidolf L. Comparison of inhibitory effects of the proton pump-inhibiting drugs omeprazole, esomeprazole, lansoprazole, pantoprazole, and rabeprazole on human cytochrome p450 activities. Drug Metab Dispos 2004; 32: 821 -827.

7. Furuta T, Iwaki T, Umemura K. Influences of different proton pump inhibitors on the anti-platelet function of clopidogrel in relation to cyp2c19 genotypes. Br J Clin Pharmacol 2010; 70: 383-392.

8. Yamane K, Kato Y, Tazaki J, Tada T, Makiyama T, Imai M, et al. Effects of PPIs and an $\mathrm{H} 2$ blocker on the antiplatelet function of clopidogrel in Japanese patients under dual antiplatelet therapy. $J$ Atheroscler Thromb 2012; 19: 559-569.

9. Bhatt DL, Cryer BL, Contant CF, Cohen M, Lanas A, Schnitzer TJ, et al. Clopidogrel with or without omeprazole in coronary artery disease. N Engl J Med 2010; 363: 1909-1917.

10. O'Donoghue ML, Braunwald E, Antman EM, Murphy SA, Bates ER, Rozenman Y, et al. Pharmacodynamic effect and clinical efficacy of clopidogrel and prasugrel with or without a proton-pump inhibitor: An analysis of two randomised trials. Lancet 2009; 374: 989-997.

11. Chitose T, Hokimoto S, Oshima S, Nakao K, Fujimoto K, Miyao Y, et al. Clinical outcomes following coronary stenting in Japanese patients treated with and without proton pump inhibitor. Circ J 2012; 76: $71-78$.

12. Aihara H, Sato A, Takeyasu N, Nishina H, Hoshi T, Akiyama D, et al. Effect of individual proton pump inhibitors on cardiovascular events in patients treated with clopidogrel following coronary stenting: Results from the Ibaraki cardiac assessment study registry. Catheter Cardiovasc Interv 2012 January 10 [Epub ahead of print] [doi: $10.1002 / \mathrm{ccd} .23327]$ 